\title{
(6) OPEN ACCESS \\ On improving the use of OCT imaging for detecting glaucomatous damage
}

\author{
Donald C Hood, ${ }^{1,2}$ Ali S Raza ${ }^{1,3}$
}

'Department of Psychology, Columbia University, New York, New York, USA ${ }^{2}$ Department of

Ophthalmology, Columbia University, New York,

New York, USA

${ }^{3}$ Department of Neurobiology and Behavior, Columbia University, New York, New York, USA

\section{Correspondence to} Professor Donald C Hood, Department of Psychology, Columbia University, 406 Schermerhorn Hall, 1190 Amsterdam Avenue, MC 5501, New York, NY 10027, USA; dch3@columbia.edu

Received 25 February 2014 Revised 28 April 2014 Accepted 29 April 2014
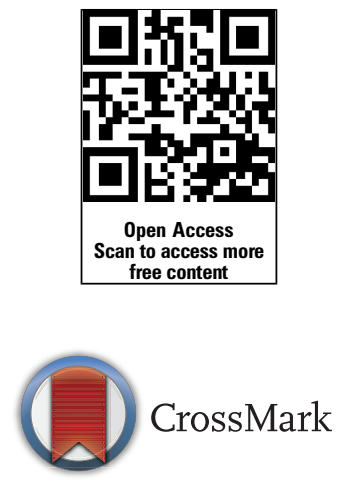

To cite: Hood DC, Raza AS. Br J Ophthalmol 2014;98: ii1-ii9.

\begin{abstract}
Aims To describe two approaches for improving the detection of glaucomatous damage seen with optical coherence tomography (OCT).
\end{abstract}

Methods The two approaches described were: one, a visual analysis of the high-quality OCT circle scans and two, a comparison of local visual field sensitivity loss to local OCT retinal ganglion cell plus inner plexiform ( $R G C+)$ and retinal nerve fibre layer (RNFL) thinning. OCT images were obtained from glaucoma patients and suspects using a spectral domain OCT machine and commercially available scanning protocols. A high-quality peripapillary circle scan (average of 50), a three-dimensional (3D) scan of the optic disc, and a 3D scan of the macula were obtained. RGC+ and RNFL thickness and probability plots were generated from the 3D scans.

Results A close visual analysis of a high-quality circle scan can help avoid both false positive and false negative errors. Similarly, to avoid these errors, the location of abnormal visual field points should be compared to regions of abnormal RGC+ and RNFL thickness.

Conclusions To improve the sensitivity and specificity of OCT imaging, high-quality images should be visually scrutinised and topographical information from visual fields and OCT scans combined.

\section{INTRODUCTION}

At one time, there was only one commercially available optical coherence tomography (OCT) machine and glaucoma specialists depended upon the summary report (figure 1A) based upon the most commonly used protocol. Numerous studies using this time-domain (td) OCT machine found that the average retinal nerve fibre layer (RNFL) thickness (arrow 1), clock hour thickness (2), and quadrant thickness (3) provide good sensitivity and specificity for detecting glaucomatous damage (see references $^{1-4}$ for reviews). These RNFL thickness measures were obtained separately from three scans and averaged for this report; the machine was too slow to average multiple images within a scan protocol. One of these scans is shown in figure $1 \mathrm{~A}$ (4) and the raw data for this same scan is enlarged and presented in grey scale in figure 1B. Given the relatively poor resolution of this peripapillary image, the success of this report is a testimonial to the robustness of these derived RNFL measures, as well as to those who developed the technique and this report. ${ }^{5-8}$

With the advent of newer technology, such as the spectral domain (sd) OCT, the quality of the images became substantially better. For example, compare the scan in figure $1 \mathrm{~B}$ to the sdOCT scan in figure 1C. The improvement in quality was partly due to improved spatial resolution, but was largely due to the averaging of multiple images within a scan (50 in the case of figure 1C) made possible by a substantially faster scan rate. In addition, because of these improvements, so-called three-dimensional (3D) scans (ie, cube or volume scans) of the regions around the disc and macula became possible. In particular, multiple lines scans can be obtained within a single scan protocol. From these images, 2D measures of the retinal ganglion cell (RGC) and RNFL thickness can be derived.

While the sdOCT allows us to see spatial detail not easily seen on the earlier tdOCT scans, our analyses have not kept pace in at least two ways. First, one of the advantages of sdOCT is that it provides topographical information about RGC and RNFL abnormalities. Thus, local RGC and RNFL loss can be topographically compared to local loss in visual field (VF) sensitivity, ${ }^{9}{ }^{10}$ as patients are routinely tested with static automated perimetry (SAP). This should improve sensitivity and specificity for detecting glaucomatous damage as SAP measurement errors should be largely independent of OCT measurement errors. Second, the improved sdOCT images allow for a direct visual analysis of the scans, much the way MRI scans are analysed, rather than depending entirely upon computer-driven summary statistics.

The purpose here is to describe two approaches for improving the detection of glaucomatous damage; one approach combines a topographical comparison of OCT and VFs and a second involves a qualitative analysis of OCT scans. These approaches are illustrated below in a one-page report.

\section{METHODS}

The data from five eyes of five patients, were used to illustrate our approach. All five had glaucomatous optic neuropathy on stereophotography evaluation and all were part of previously published studies. ${ }^{11} 12$ They had 24-2 and 10-2 VFs tests obtained with the SITA-standard protocol (Humphrey VF Analyzer; Carl Zeiss Meditec, Dublin, California, USA); for inclusion, the mean deviation (MD) on the 24-2 VF had to be better than $-6 \mathrm{~dB} .{ }^{12}$ Written informed consent was obtained from all of the participants. Procedures followed the tenets of the Declaration of Helsinki, and the protocol was approved by the institutional review board of Columbia University.

\section{OCT protocol}

A sdOCT machine (3D OCT-2000, Topcon) and the following three scan protocols were used: $6.0 \times 6.0 \mathrm{~mm} \quad 3 \mathrm{D}$ disc $(512$ A-scans by 128 B-scans); $6.0 \times 6.0 \mathrm{~mm} \mathrm{3D}$ macula (512 A-scans by $128 \mathrm{~B}$-scans); and $3.4 \mathrm{~mm}$ dia. circle (average of 50 scans; 1024 A-scans). The circle protocol involved 
Figure 1 Peripapillary retinal nerve fibre layer data from Patient 1 , showing (A) the report from the Zeiss Stratus time-domain optical coherence tomography (tdOCT) machine based on the average of 3 scans for both eyes (RNFL Thickness (3.4) protocol), (B) the TSNIT circle scan path on top of infrared fundus (left) and a single raw circle tdOCT image (right) for the right eye, (C) the sdOCT TSNIT circle scan path on top of fundus (left) and averaged circle sdOCT image (right) for the same eye, and (D) the sdOCT NSTIN circle scan path (left) and averaged circle sdOCT image (right).
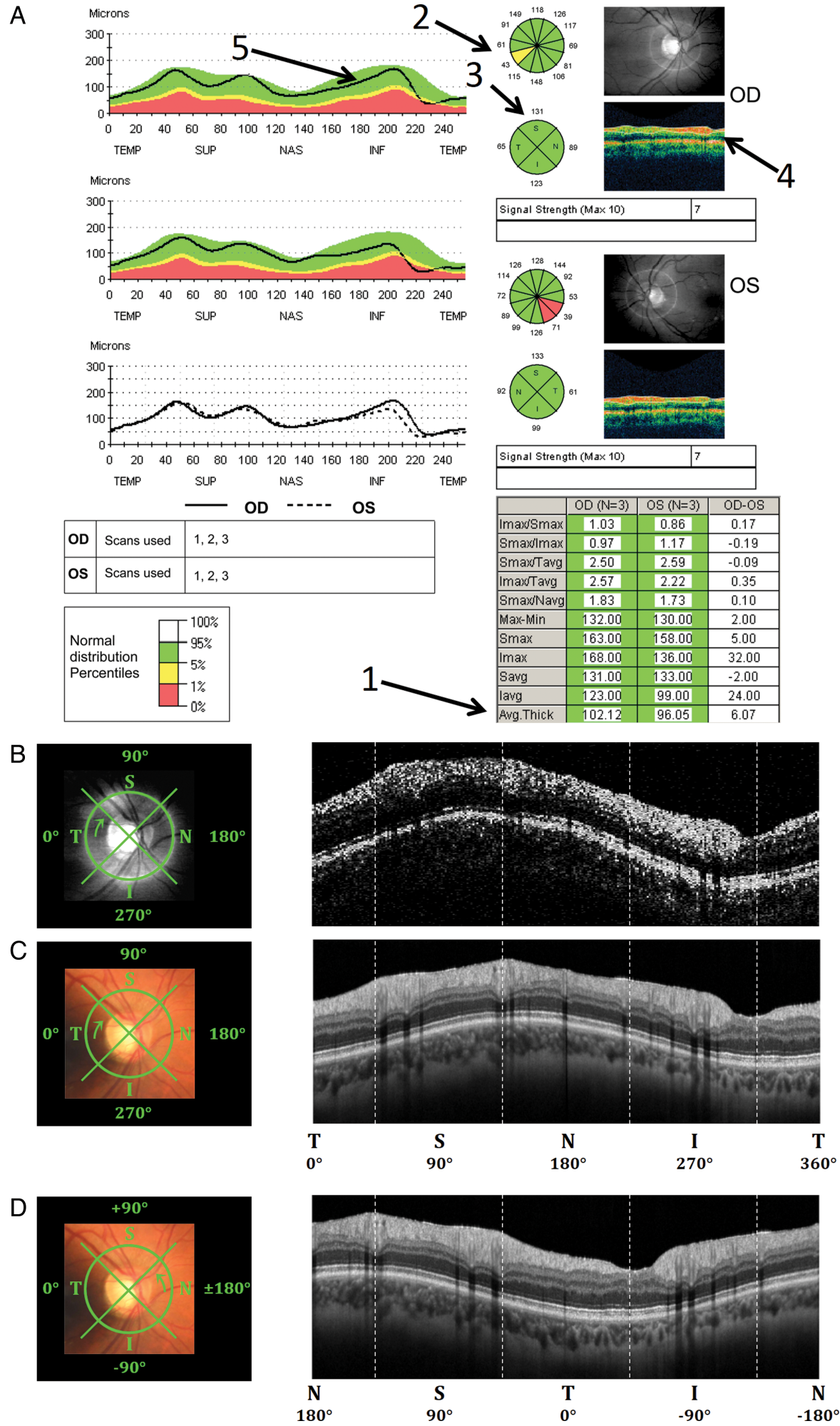

averaging 50 individual scans and thus produced an image of relatively high-quality. (Note that shadowgrams (see inset in panel $\mathrm{E}$ of figures 3 (green arrow), 5 and 6) can be examined for artefacts such as excessive eye movements, blinks, etc.)

\section{OCT analysis}

Peripapillary RNFL analysis

The high-quality peripapillary image produced by the circle scan was qualitatively analysed as described in the Results. In addition, this image was segmented by the machine's software and a RNFL thickness plot produced. While the circle scan has the advantage of being of high quality, it can be off-centre if the patient has considerable trouble fixating or the operator is not careful. The fundus photo with the superimposed scan location (inset in panel A of figures 2, 3, 5 and 6) provides some information about how well centred the scan was.

The commercial software also derives a peripapillary RNFL thickness plot from the segmentation of the $3 \mathrm{D}$ disc scan, by centring a circle after the scan is obtained. To distinguish this plot from the one based upon the high-quality averaged circle 
Figure 2 Visual field (VF) and spectral domain optical coherence tomography (sdOCT) data for Patient 2 along with models relating VF locations to OCT, all shown as if for right eye. (A) The NSTIN averaged circle sdOCT image with computer-derived retinal nerve fibre layer (RNFL) segmentation (green lines) and corresponding circle scan path on top of fundus (inset). (B) The averaged circle RNFL thickness (grey dashed line) calculated based on the segmentation in (A) and the extracted circle RNFL thickness (solid black line), with regions corresponding to the superior (magenta) and inferior (dark blue) macula in unique colours. Both RNFL thickness plots are superimposed on coloured regions indicating the $95 \%$ to $5 \%$ (green), $5 \%$ to $1 \%$ (yellow), and less than 1\% (red) ranges of normative data. The red vertical lines indicate the average location of the major blood vessels in a group of patients. (C) The 24-2 VF for the same patient with (D) a model relating the locations of the VF to regions of the circle scans. (E) The 10-2 VF for the same patient with (F) VF to regions of the circle scans. The bold dark blue line indicates the macular vulnerability zone (MVZ). a model relating the locations of the

A

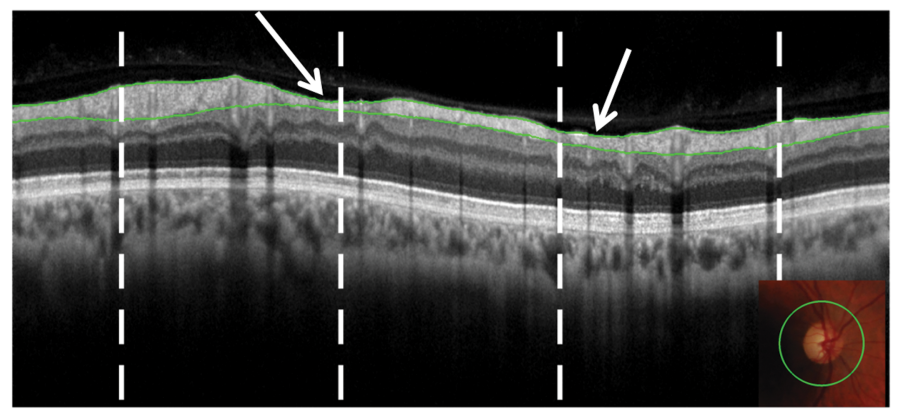

B
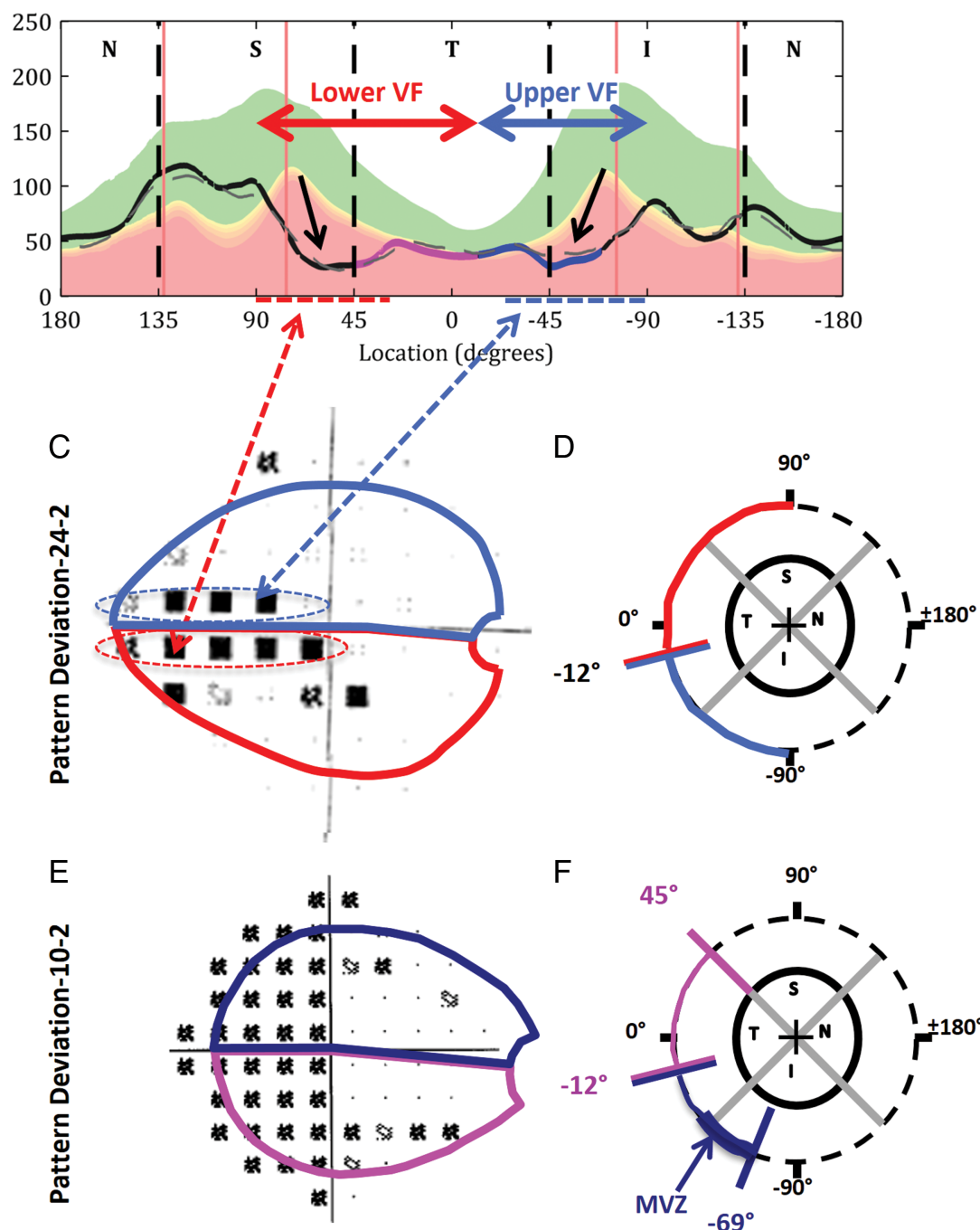

scan, it will be called the 'extracted circle RNFL plot'. (Note: As technological advances improve the quality of the 3D scan, the circle scan can be replaced by an image of the peripapillary circle extracted from the 3D scan.)

\section{D RNFL and RGC+ thickness maps}

As previously described, ${ }^{9}$ RGC + and RNFL thickness maps were derived from the macular and disc $3 \mathrm{D}$ scans and turned into probability plots by comparing the thicknesses to normative values.

\section{RESULTS}

Below we describe elements of a report for detecting RGC/ RNFL damage based upon sdOCT imaging. The report is designed to illustrate the power of combining VF and OCT information, as well as the value-added in carefully examining the OCT images.

Reports should display RNFL thickness plots as NSTIN, not TSNIT plots

In the original tdOCT report, the peripapillary circle scan (arrow 4 in figure 1A) was presented with the nasal quadrant $(\mathrm{N})$ in the middle and the temporal $(\mathrm{T})$ quadrant divided between the left and right ends. The accompanying RNFL thickness plot (arrow 5 in figure 1A) has been called the 'TSNIT plot'. Instead, we recommend using a 'NSTIN plot', in which the most important portion of the disc for visual function, the temporal quadrant, is in the middle (figure 1D). As illustrated 
A

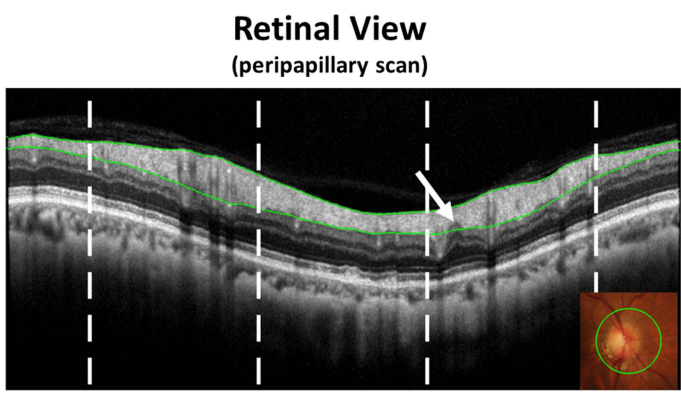

B

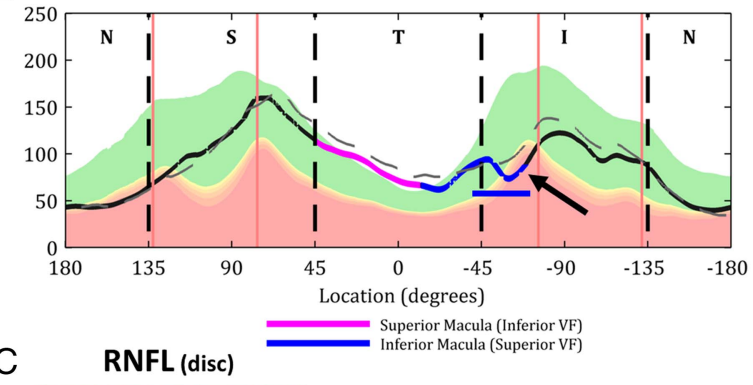

C

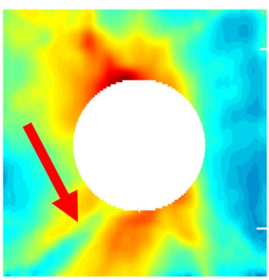

D

RNFL (macula)

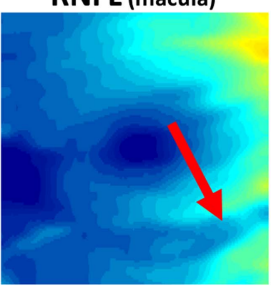

\section{Retinal View}

(thickness plots)

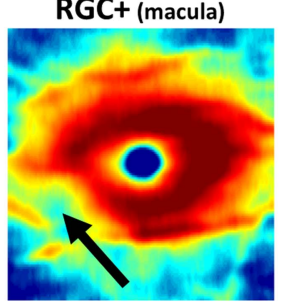

E

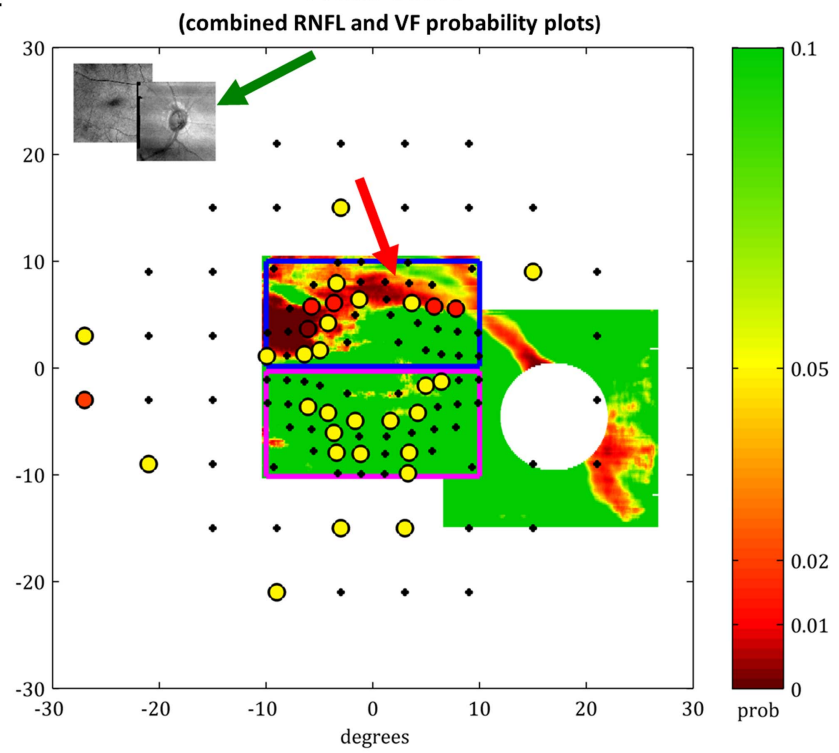

F

Field View

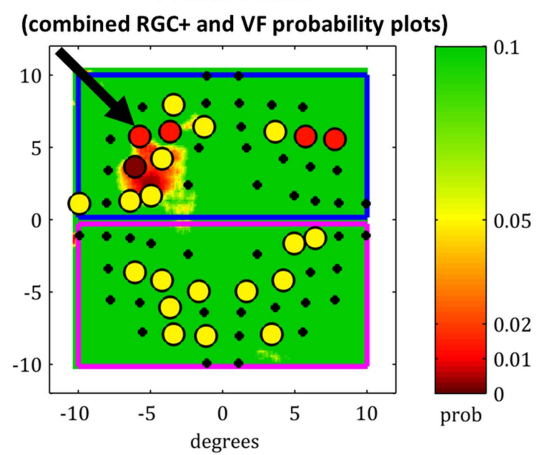

Figure 3 The single-page spectral domain optical coherence tomography report for Patient 3 showing (A) an enlarged averaged circle scan in NSTIN view as in figure 2A, (B) the corresponding retinal nerve fibre layer (RNFL) thickness as in figure 2B, (C) the 2D RNFL thickness from the 3D disc scan, (D) the 2D RNFL (left) and retinal ganglion cell (RGC)+ (right) thicknesses from the 3D macular scan, (E) the co-registered macular and disc 2D RNFL thickness probability plots in field view with the 24-2 and 10-2 visual field (VF) test point probabilities superimposed (see colour bar) with an inset of the co-registered shadowgrams of the 3D scans (green arrow), and (F) the macular 2D RGC+ thickness probability plot in field view with the 10-2 VF test point probabilities superimposed (see colour bar) as in (E).

below, this allows for easier visualisation of the relationship between RNFL thinning and VF defects.

\section{Reports should contain an enlarged image of the circle scan}

Figure 2A shows the averaged circle scan from Patient 2 with the computer-derived borders of the RNFL in green. There are two reasons for including an image larger than seen in the tdOCT report in figure 1A (arrow 4). First, the validity of the computer-marked borders can be assessed. All current OCT computer algorithms make mistakes (segmentation errors), which will be typically overlooked if the scan image is presented as a small inset as in figure 1A. Epiretinal membranes, vitreous detachments, and poor image contrast can lead to segmentation errors and thus errors in the associated RNFL thickness measures. The enlarged, averaged circle scan has sufficient detail so that these errors can be identified.

Second, the high-quality image allows for a qualitative assessment of the details of the scan. For example, in figure $2 \mathrm{~A}$ local
RNFL thinning can be seen in two regions (see white arrows). Other details of interest can be seen in some circle scans as illustrated below.

\section{Look for regions of abnormal thinning on the RNFL plot and compare to scan}

After scrutinising the averaged circle scan image, the RNFL thickness plots should be examined. The dashed and solid curves in figure $2 \mathrm{~B}$ are the RNFL thickness plots from the averaged circle and 3D scans, respectively. If these curves agree and the segmentation seen on the scan appears accurate, these plots can be trusted. If they disagree, it is usually possible to decide why.

For example, the averaged circle (dashed) and extracted circle (solid) RNFL thickness plots from Patient 3 in figure 3B disagree in two locations. First, the thickness in the temporal quadrant and part of the superior quadrant is slightly greater in the case of the averaged circle, probably because the circle scan is off- 


\section{A Patient 3}
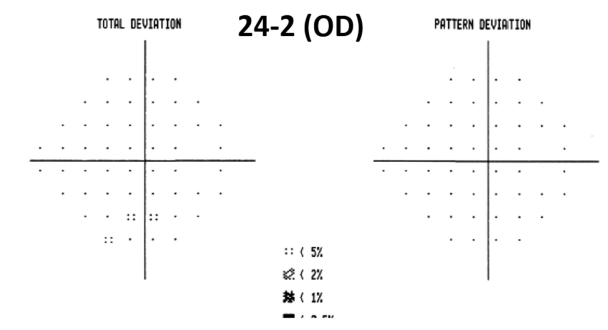

\section{Patient 4}

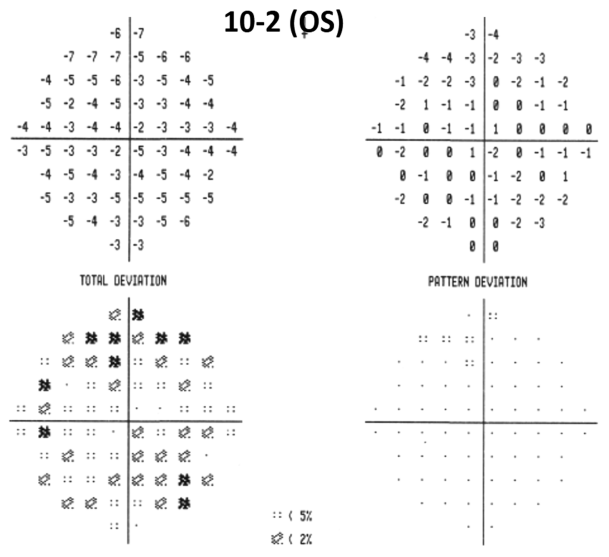

D Patient 5

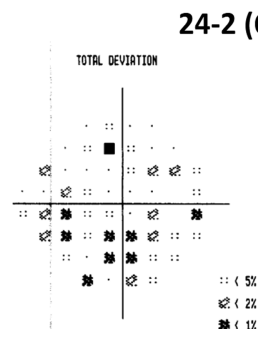

\section{B Patient 3}

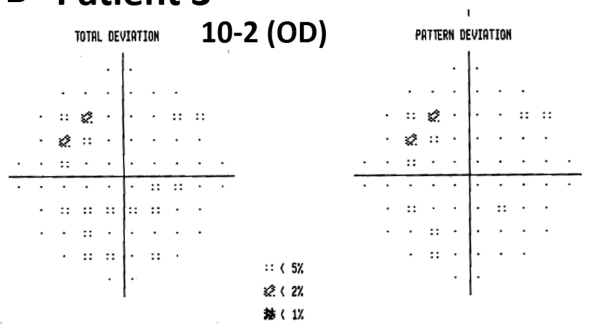

\section{E Patient 5}

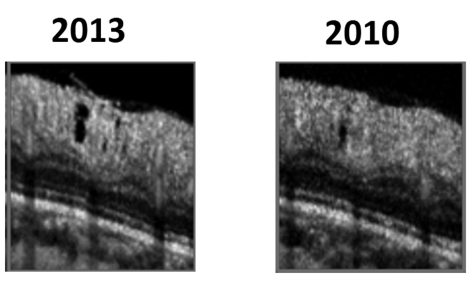

Figure 4 (A) The 24-2 and (B) 10-2 visual field (VF) data for Patient 3. (C) The 10-2 VF data for Patient 4, (D) the 24-2 VF data from 2013 for Patient 5, and (E) enlargements of a hole observed in the averaged circle scan of Patient 5 in both 2013 (left) and 2010 (right).

centre; see the fundus photograph (inset in panel A). Second, and more important, there is a suggestion of a small local defect on the extracted circle RNFL plot (solid), but not on the averaged circle plot (dashed). A close examination of the scan in panel A, indicates a small, local thinning, which the algorithm appears to underestimate on the averaged circle scan. This is discussed further below.

After assessing the veracity of the RNFL plot (figure $2 \mathrm{~B}$ ), the regions of abnormal thinning can be identified. In this case, the RNFL plot falls well below the $99 \%$ confidence limit in the same regions (black arrows in figure $2 \mathrm{~B}$ ) that show local thinning on the scan in figure $2 \mathrm{~A}$ (white arrows).

\section{Examine the general topographical agreement between peripapillary RNFL thinning and loss in VF sensitivity}

With a little practice, the topographical agreement between peripapillary RNFL thinning and VF defects can be assessed qualitatively. To make this assessment, one needs to take into consideration the relationship between regions of the disc and regions of the VF.

Figure 2 illustrates this relationship. The 24-2 pattern deviation (PSD) plot for this eye is shown in panel C. The red and light blue arrows in panel B mark the temporal half of the circle scan, that is, they extend from $+90^{\circ}$ to $-90^{\circ}$ as shown in panel D. The corresponding region of the 24-2 VF is enclosed within the light blue and red contours in panel C. These contours were drawn to be in general agreement with previous work. $^{13}{ }^{14}$ While these contours are approximate, and will differ somewhat among individuals, ${ }^{13}{ }^{15}$ they are useful for deciding whether there is agreement among the OCT and VF findings.

The dashed red and blue horizontal lines along the $\mathrm{x}$-axis in figure $2 \mathrm{~B}$ show the regions of the RNFL plot that correspond to the abnormal regions of the VF (ie, the points falling within the dashed ellipses in C). In this case there is excellent agreement.

The RNFL plot also suggests there is damage in the macula, defined here as the central $\pm 8^{\circ}$. To facilitate the detection of macular damage on the RNFL plots, the regions of the scan associated with the RGC fibres from the central $\pm 8^{\circ}$ retina are coded as magenta (superior retina/lower VF) and dark blue (inferior retina/upper VF). These boundaries (see panel F), as well as the corresponding regions on the 10-2 (panel E), are based upon a recent map of the macula. ${ }^{16}$ Note that macular damage is confirmed by the $10-2 \mathrm{VF}$ in panel $\mathrm{E}$. 
A

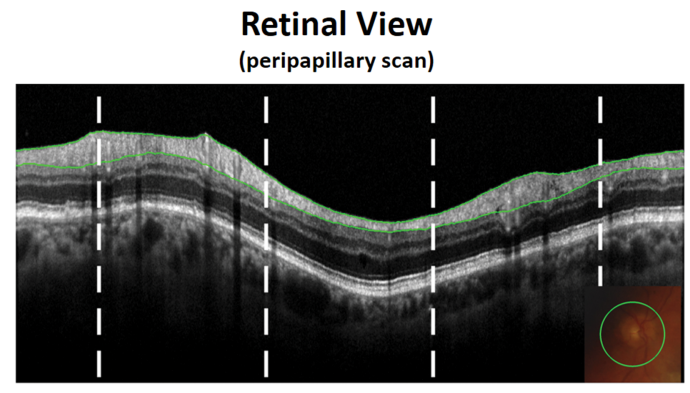

B

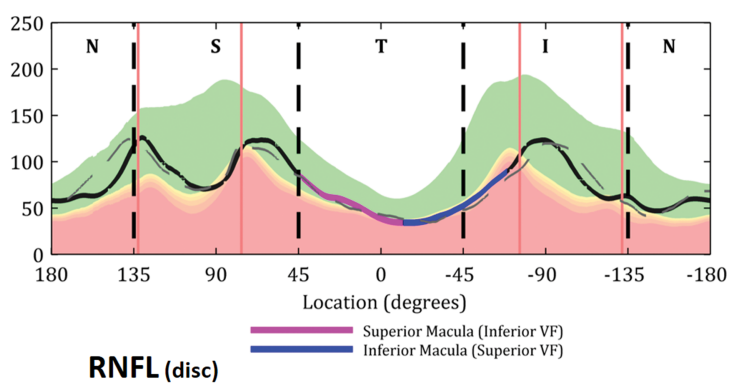

C

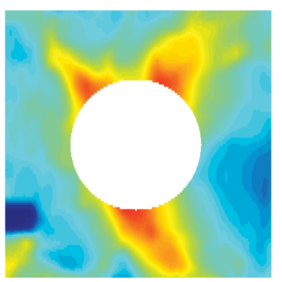

D

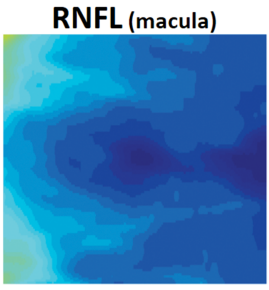

\section{Retinal View}

(thickness plots)

RGC+ (macula)

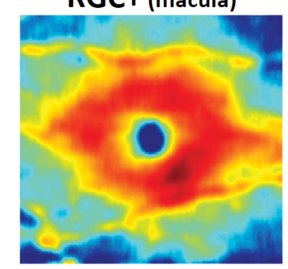

$\mathrm{E}$

Field View

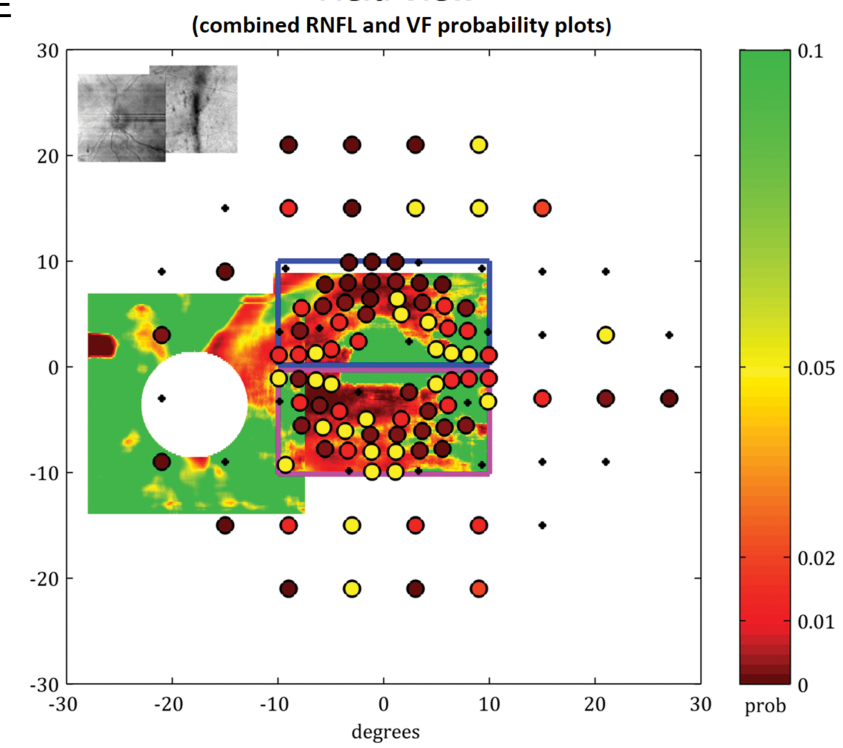

Field View

$\mathrm{F}$

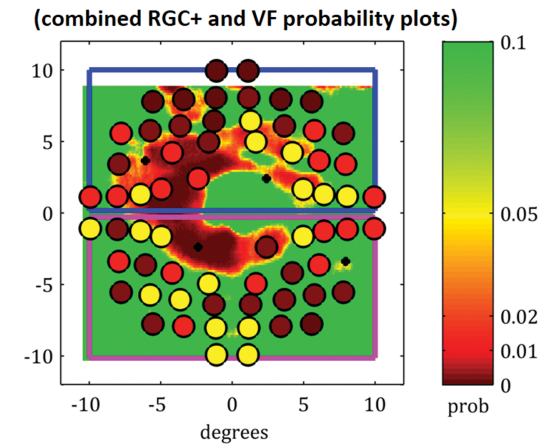

Figure 5 The single-page spectral domain optical coherence tomography report for Patient 4 as in figure 3 showing diffuse retinal ganglion cell $(\mathrm{RGC})+$ thinning (panel D, right). The agreement of the RGC+ (panel F) and retinal nerve fibre layer (RNFL) (panel E) probability plots with the visual field (VF) probability plot suggests diffuse macular damage.

Look for macular damage especially in the macular vulnerability zone

We have identified two types of macular damage on OCT scans. ${ }^{12}$ One type, considered below, is diffuse/widespread and is typically relatively shallow and difficult to identify with RNFL scans. The second tends to be deeper and to occur in a relatively narrow portion of the inferior disc labelled macular vulnerability zone (MVZ) in figure $2 \mathrm{~F}$.

This local macular damage easily can be missed if one does not look closely at the high-quality averaged circle scan and the RNFL plots. Figure 3A,B shows what appears to be a relatively normal RNFL thickness plot, although there is probably local nasal thinning far outside the 24-2 VF. The commercial report (not shown) indicated normal RNFL thickness in the inferior, temporal and superior quadrants and the associated clock hours. The 24-2 VF (figure 4A) appeared normal as well with a MD of $-1.50 \mathrm{~dB}$, a PSD of $1.16 \mathrm{~dB}$, and a glaucoma hemifield test (GHT) within normal limits. However, as mentioned above, the extracted circle RNFL plot from the 3D scan (solid curve in figure $3 \mathrm{~B}$ ) shows a very local abnormal region (arrow). Given the VF results, this local abnormality could easily be overlooked. In any case, the scan in figure 3A shows a small local thinning (white arrow). This local thinning is in the middle of the MVZ, which is shown as the horizontal dark blue line. The damage in the MVZ can be missed on 24-2 VF tests because they poorly sample the region of glaucomatous damage of the macula seen on OCT. ${ }^{16}$

The patient's 10-2 VF (figure 4B) shows a subtle defect in the general region one would expect from the model of macular damage. ${ }^{16}$ However, given the subtle nature of both the OCT and $\mathrm{VF}$ changes, there is reason for scepticism. Figure 3 is our one page report of RGC/RNFL damage; the other panels, described below, confirm the damage is real.

\section{Examine the RGC layer thickness maps obtained from the macular 3D scan}

RGC thickness maps are useful for identifying macular damage, which can be missed on peripapillary RNFL analysis. In general, the commercial machines supply an analysis of macular 3D scans that includes RGC thickness. In some cases, this is the thickness of the combined RGC, inner plexiform layer (IPL) and RNFL, while in others it is the thickness of the combined RGC+IPL (called here RGC+). Figure 3D (right panel) shows the patient's RGC+ thickness derived from the macular 3D scan and displayed in pseudo-colour. By 
A
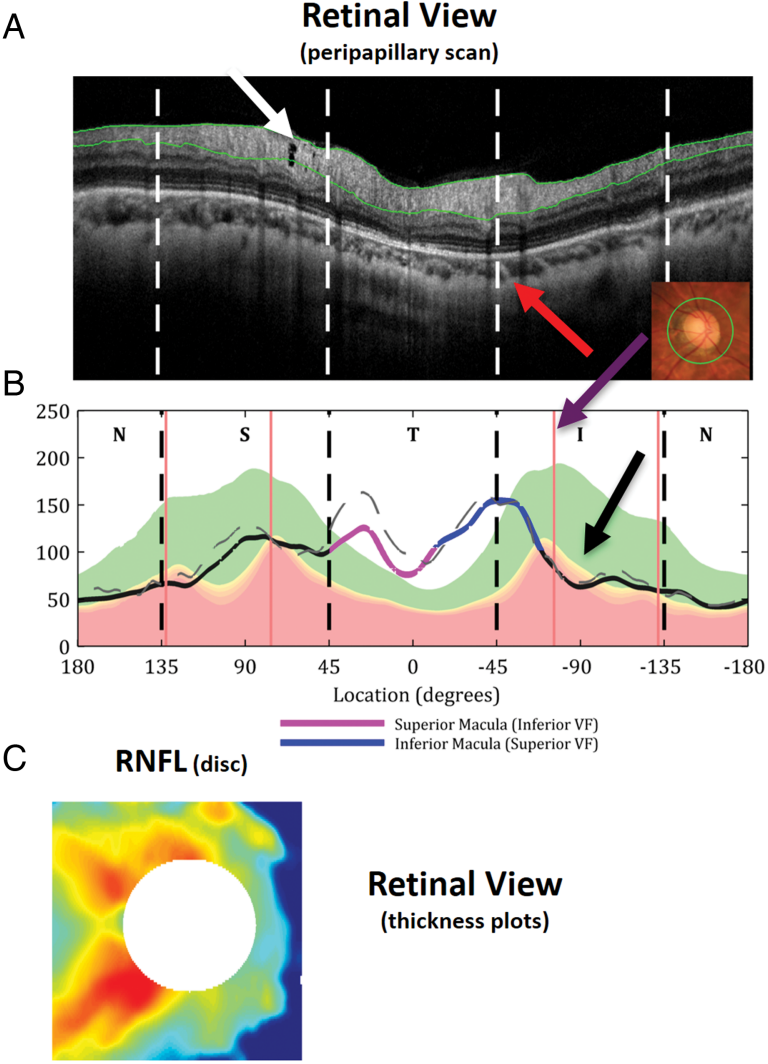

D

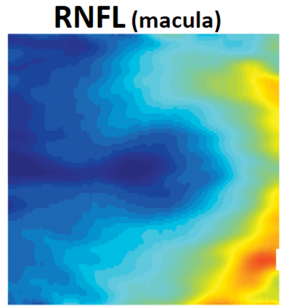

Retinal View

(thickness plots)
$\mathrm{E}$

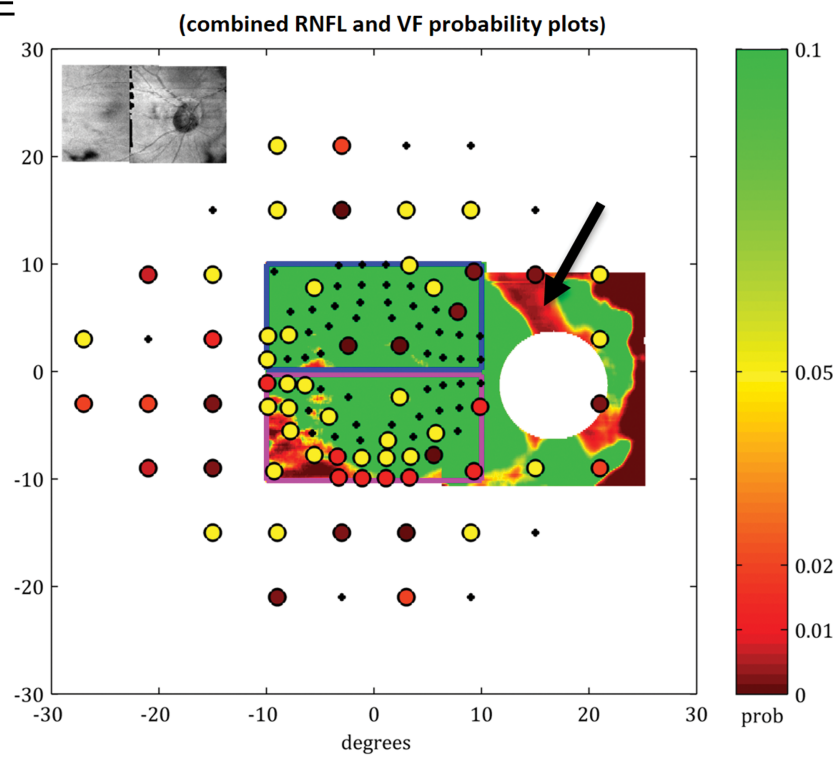

Field View

$\mathrm{F}$

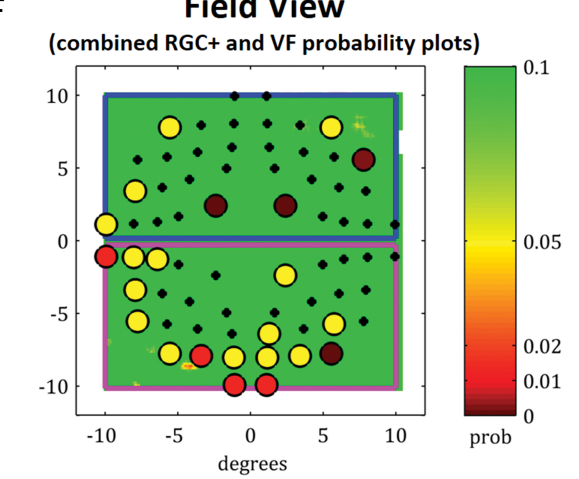

Figure 6 The single-page spectral domain optical coherence tomography report for Patient 5 as in figure 3 illustrating the need for a close scrutiny of peripapillary scans (panel A). The peripapillary retinal nerve fibre layer (RNFL) thickness plot (panel B) and the RNFL probability plot (panel E) suggest an abnormally thin RNFL associated with the upper visual field. However, this is probably a false positive, as indicated by a close examination of the location of the inferior temporal blood vessels (red arrow in panel A) in this eye to the average location in a group of patients (purple arrow in panel B), as well as the abnormally thick RNFL in the temporal quadrant. The repeat visual fields (VFs) are consistent with this interpretation. On the other hand, the presence of a hole (black arrow in panel A) suggests that there may be very early glaucomatous damage in the superior disc.

comparing the local thickness values to healthy controls, a probability plot can be created as shown in figure 3F. This probability plot is displayed in field view. The abnormally thin RGC+ layer (black arrows in figure 3D,F) argues for macular damage in this eye.

\section{Examine the RNFL layer thickness maps obtained from} macular and disc 3D scans

From the 3D scans of the disc most commercial machines produce RNFL thickness plots as shown in figure 3C. A RNFL thickness plot can also be produced for the macular 3D scans as shown in figure 3D (left). By comparing the thickness values to healthy controls, this thickness plot can be turned into a probability plot. For the report, the RNFL probability plots from the macular and disc 3D scans are combined by aligning the blood vessels and then displayed in field view (figure 3E). The local macular damage is clearly apparent in this RNFL thickness analysis as indicated by the red arrows in panels C, D (left) and $\mathrm{E}$.

Examine the topographical agreement between local RNFL and RGC+ thinning and local loss in VF sensitivity

The best way to assess the topographical agreement between RNFL thinning and VF sensitivity loss is to superimpose the probability plots for each as previously described. ${ }^{9}$ This analysis is shown on the right side of our report.

Figure $3 \mathrm{~F}$ shows the points from this patient's 10-2 VF (figure 4B) superimposed on the field view of the RGC+ probability plot. The significance levels of the 10-2 points and RGC + thickness are colour-coded using the same continuous probability scale. To make this comparison, the locations of the VF points need to be adjusted to account for the displacement of the RGCs in the fovea. ${ }^{9} 10$

In a similar manner, in figure $3 \mathrm{E}$ the points of the $24-2$ and 10-2 VF are superimposed upon the RNFL probability plot 
(field view) and coded with the same continuous probability scale. There is good local agreement between the OCT and VF results for the upper $\mathrm{VF} /$ inferior retina.

While it may be difficult for manufacturers to incorporate VF data into their software, it should be relatively easy for them to indicate the 24-2 and 10-2 locations on the OCT probability plots and leave it to the clinician to circle the VF points that are abnormal.

\section{Using the report to detect diffuse macular damage}

The report for Patient 4 in figure 5 illustrates an example of what we believe is diffuse macular damage. ${ }^{12}$ This patient's 10-2 VF (figure 4C) had a MD of $-4.15 \mathrm{~dB}(\mathrm{p}<1 \%)$ and the total deviation values showed a relatively homogeneous loss across the VF. Given that the PSD was normal, many would attribute this diffuse loss of sensitivity to non-glaucomatous factors. The peripapillary RNFL plot in figure $5 \mathrm{~B}$ is also ambiguous with the region corresponding to the macula (magenta and dark blue) falling in the normal (green) or borderline (yellow) region. The comparison of RGC+ and VF abnormalities in panel $\mathrm{F}$, however, suggests diffuse macular damage. The RNFL and VF comparison in panel E agrees. On exam, this patient did not have cataracts, but did have a best corrected visual acuity (BCVA) of 20/50, also consistent with diffuse damage.

\section{Carefully examine the actual scan images}

There is much to be learned by carefully examining the actual OCT scan images. Below, we illustrate three examples.

\section{False positives and the location of blood vessels}

Figure 6 shows our report for the right eye of Patient 5. A glaucoma specialist noted peripapillary atrophy and diffuse thinning on fundus stereo photograph. The report from the commercial machine showed abnormal RNFL thinning in the inferior quadrant. Our report appeared to confirm this finding (black arrows in figure 6B). However, while the OCT analysis was consistent with local damage, the fundus exam and VF (figure 4D) were consistent with diffuse damage. On closer analysis, we concluded that the OCT result was a false positive, an example of what has been called 'red disease'. ${ }^{17}$ Notice the abnormally thick RNFL in the temporal quadrant in figure 6A,B. The major blood vessels (BVs) in this eye are located more temporally than the average location for the controls. The red lines in panel B show the average locations of the major four groups of BVs for a group of patients. ${ }^{18}$ The red arrow in panel $\mathrm{A}$ indicates the location of the inferior-temporal (IT) BVs in this eye, while the purple arrow shows the average location of the IT BVs. As expected, the location of the IT BVs correspond to the location of the inferior peak of the RNFL plot (peak of green in panel A) in healthy controls. ${ }^{19}$ This is, in part, due to the direct contribution of the BVs to the RNFL thickness and in part due to the fact that the thickest region of RNFL tends to be close to the major BVs. ${ }^{19}$ In any case, the location of the IT BVs in this eye is probably the reason for the abnormally thick RNFL in the temporal quadrant and the abnormally thin RNFL in the inferior quadrant. The 24-2 VF is probably a false positive as well. Note that the 24-2 test from 2012, 1 year earlier, was normal, while the 24-2 VF obtained 2 years earlier showed diffuse loss.

\section{Hypodense (holes)}

While the inferior quadrant of the RNFL profile of this eye illustrates a false positive OCT result, the scan in figure 6A has evidence of local damage in the superior quadrant of the disc.
In particular, there is a hypodense region (white arrow in figure 6A). We have shown that these holes are actually tunnels that follow an arcuate pattern and are almost surely very local, and very small, RNF bundle defects. ${ }^{20}$ We have only seen them in patients with glaucoma or patients who are glaucoma suspects. $^{21}$ They also show progression as seen for this eye in figure $4 \mathrm{E}$.

\section{Examine horizontal and vertical macular scans for outer retinal damage}

Finally, we routinely examine the $3 \mathrm{D}$ macular scans, as well as a high-quality horizontal line scans through the macula, for signs of outer retinal damage. This is particularly important in eyes with reduced visual acuity. It is common to see previously unnoticed signs of epiretinal membranes, age-related macular degeneration, oedema, and macular holes in glaucoma patients and suspects. ${ }^{22}$

\section{DISCUSSION}

While once there was a single OCT report used to identify glaucomatous damage, now every commercial machine offers more than one glaucoma report. However, by and large, these reports fail to take full advantage of the spatial detail available. We argued above that the effectiveness of the OCT could be improved by a qualitative analysis of enlarged, high-quality images and by a topographical comparison of the abnormal regions seen on OCT to those seen on VFs. Our one-page report was developed to incorporate this information.

However, no one report will suffice. The high-quality OCT scans have all the complexities of MRI scans, as well as better spatial resolution. Yet, MRI scans are not analysed by computer algorithms and summarised in simple summary statistics and diagrams, as is typically the case with OCT scans. In fact, relying solely on summary statistics plays a major role in apparent disagreements between the results of VF and OCT tests. ${ }^{23}$ In the case of MRI scans, radiologists read and interpret the scans for other specialists, while in the case of OCT scans, ophthalmologists are left to interpret the scans on their own.

While the report developed here is meant to help ophthalmologists in this interpretation, it will not suffice. On one hand, the summary statistics and diagrams seen in figure 1A are still of use and new summary statistics combining VF and OCT information are being developed. ${ }^{24-30}$ The sensitivity and specificity of these various summary statistics need to be compared to alternative analyses, such as the report describe here with and without summary statistics. On the other hand, the full power of the OCT will rely on a careful analysis of high-quality images performed by individuals trained to read these scans, much the way a radiologist reads an MRI. This will be increasingly the case as OCT image quality continues to improve. Admittedly, for many cases a simple report or summary statistic may do. However, for difficult and ambiguous cases, the non-specialist may need to rely on a member of their department/group who has considerable experience analysing OCT scans.

Acknowledgements We thank Paula Alhadeff, Dana Blumberg, and Jeffrey Liebmann for their comments and advice, and especially Drs Robert Ritch, Jeffrey Odel and Gustavo de Moraes for their help and support throughout this project.

Contributors Both authors were responsible for the design of the study and interpretation of the data, as well as preparation, review and approval of the article.

Funding The study was supported by National Institutes of Health Grant R01-EY-02115 and a grant from Topcon, Inc.

Competing interests The study was supported by a grant from Topcon, Inc. Patient consent Obtained.

Ethics approval The Institutional Review Board of Columbia University. 
Provenance and peer review Commissioned; externally peer reviewed.

Open Access This is an Open Access article distributed in accordance with the Creative Commons Attribution Non Commercial (CC BY-NC 3.0) license, which permits others to distribute, remix, adapt, build upon this work non-commercially, and license their derivative works on different terms, provided the original work is properly cited and the use is non-commercial. See: http://creativecommons.org/ licenses/by-nc/3.0/

\section{REFERENCES}

1 Zangwill LM, Bowd C. Retinal nerve fiber layer analysis in the diagnosis of glaucoma. Curr Opin Ophthalmol 2006;17:120-31.

2 Lin SC, Singh K, Jampel HD, et al. Optic nerve head and retinal nerve fiber layer analysis: a report by the American Academy of Ophthalmology. Ophthalmology 2007;114:1937-49

3 Chang R, Budenz DL. New developments in optical coherence tomography for glaucoma. Curr Opin Ophthalmol 2008;19:127-35.

4 Sharma P, Sample PA, Zangwill LM, et al. Diagnostic tools for glaucoma detection and management. Surv Ophthalmol 2008;53:17-32.

5 Huang D, Swanson EA, Lin CP, et al. Optical coherence tomography. Science 1991:254:1178-81.

6 Swanson EA, Izatt JA, Hee MR, et al. In vivo retinal imaging by optical coherence tomography. Opt Lett 1993;18:1864-6.

7 Schuman JS, Hee MR, Puliafito CA, et al. Quantification of nerve fiber layer thickness in normal and glaucomatous eyes using optical coherence tomography. Arch Ophthalmol 1995;113:586-96.

8 Schuman JS, Pedut-Kloizman T, Hertzmark E, et al. Reproducibility of nerve fiber layer thickness measurements using optical coherence tomography. Ophthalmology 1996;103:1889-98.

9 Hood DC, Raza AS. Method for comparing visual field defects to local RNFL and RGC damage seen on frequency domain OCT in patients with glaucoma. Biomed Opt Exp 2011:2:1097-105.

10 Raza AS, Cho JS, de Moraes CGV, et al. Retinal ganglion cell layer thickness and local visual field sensitivity in glaucoma. Arch Ophthalmol 2011;129:1529-36.

11 Hood DC, Raza AS, de Moraes CGV, et al. Initial arcuate defects within the central 10 degrees in glaucoma. Invest Ophthalmol Vis Sci 2011;52:940-6.

12 Hood DC, Slobodnick A, Raza AS, et al. Early glaucoma involves both deep local, and shallow widespread, retinal nerve fiber damage of the macular region. Invest Ophthalmol Vis Sci 2014;55:632-49.

13 Garway-Heath DF, Poinoosawmy D, Fitzke FW, et al. Mapping the visual field to the optic disc in normal tension glaucoma eyes. Ophthalmology 2000;107:1809-15.

14 Jansonius NM, Nevalainen J, Selig B, et al. A mathematical description of nerve fiber bundle trajectories and their variability in the human retina. Vis Res 2009:49:2157-63.
15 Hood DC, Anderson SC, Wall M, et al. A test of a linear model of glaucomatous structure-function loss reveals sources of variability in retinal nerve fiber and visual field measurements. Invest Ophthalmol Vis Sci 2009:127:875-81.

16 Hood DC, Raza AS, de Moraes CGV, et al. Glaucomatous damage of the macula. Prog Retin Eye Res 2013:32:1-21.

17 Chong GT, Lee RK. Glaucoma versus red disease: imaging and glaucoma diagnosis. Curr Opin Ophthalmol 2012;23:79-88

18 Hood DC, Wang DL, Raza AS, et al. The locations of circumpapillary glaucomatous defects seen on frequency-domain OCT scans. Invest Ophthalmol Vis Sci 2013:54:7338-43.

19 Hood DC, Fortune B, Arthur SN, et al. Blood vessel contributions to retinal nerve fiber layer thickness profiles measured with optical coherence tomography. J Glaucoma 2008;17:519-28.

20 Xin D, Talamini CL, Raza AS, et al. Hypodense regions ("holes") in the retinal nerve fiber layer in frequency-domain OCT scans of glaucoma patients and suspects. Invest Ophthalmol Vis Sci 2011:52:7180-6.

21 Xin D, Fernandes D, Nguyen M, et al. Hypodense regions ("holes") are seen in the retinal nerve fiber layer of the frequency-domain OCT scans of eyes with glaucoma, but not eyes with MS or ION. Invest Ophthalmol Vis Sci 2012;53:ARVO E-Abstract 3914.

22 Liebmann JM, de Moraes CGV, Fernandes DB, et al. Glaucoma patients with clinically normal macular appearance may have retinal abnormalities. Invest Ophthalmol Vis Sci 2012;53:ARVO E-Abstract 1001.

23 De Moraes CGV, Liebmann JM, Ritch $R$, et al. Understanding disparities among diagnostic technologies in glaucoma. Arch Ophthalmol 2012;130:833-40.

24 Brigatti L, Hoffman D, Caprioli J. Neural networks to identify glaucoma with structural and functional measurements. Am J Ophthalmol 1996;121: 511-21

25 Bowd C, Hao J, Tavares IM, et al. Bayesian machine learning classifiers for combining structural and functional measurements to classify healthy and glaucomatous eyes. Invest Ophthalmol Vis Sci 2008;49:945-53.

26 Racette $\mathrm{L}$, Chiou $\mathrm{CY}$, Hao J, et al. Combining functional and structural tests improves the diagnostic accuracy of relevance vector machine classifiers. J Glaucoma 2010;19:167-75.

27 Bizios D, Heijl A, Bengtsson B. Integration and fusion of standard automated perimetry and optical coherence tomography data for improved automated glaucoma diagnostics. BMC Ophthalmol 2011;11:20.

28 Boland MV, Quigley HA. Evaluation of a combined index of optic nerve structure and function for glaucoma diagnosis. BMC Ophthalmol 2011;11:6.

29 Medeiros FA, Lisboa R, Weinreb RN, et al. A combined index of structure and function for staging glaucomatous damage. Arch Ophthalmol 2012:130:1107.

30 Raza AS, Zhang X, De Moraes CG, et al. Improving glaucoma detection using spatially correspondent clusters of damage and by combining standard automated perimetry and optical coherence tomography. Invest Ophthalmol Vis Sc 2014;55:612-24. 